\title{
EL PROBLEMA DE LA NOSOGRAFÍA EN LA OBRA PSIQUIÁTRICA DE J. GINÉ Y PARTAGÁS
}

\author{
Antonio Diéguez Gómez \\ Psiquiatra - C.S.M. - c/ Mar Caspio, 8 - Hortaleza. Madrid (España)
}

A Beatriz

\section{RESUMEN}

Se analizan las características más relevantes del pensamiento nosográfico de Giné y Partagás y se plantean algunos interrogantes acerca de anteriores consideraciones tópicas sobre su obra. Por imperativos de la clínica Giné adopta una actitud pragmática, relativizando su militancia positivista y presentando un planteamiento nosográfico ecléctico. Debido a la adscripción unitarista de algunos de los más importantes inspiradores de su obra, esta doctrina quedaría asumida implícitamente en la misma.

\section{SUMMARY}

The most relevant characteristics of the Giné y Partagás's nosographic thinking are analyzed, and some questions about the earlier topical considerations on his work are arised. Pressed by the clinic, Giné adopts a pragmatic attitude, giving a relative importance to his positivist militancy and offering an eclectic nosographic approach. Because the unitarist attachment of some of de most important authors inspiring his work, this doctrine would remain implicitly assumed in itself.

\section{INTRODUCCIÓN}

La historiografía de la psiquiatría española acostumbra a señalar, con respecto a la labor realizada por Giné y Partagás, varios hitos que marcan en su conjunto el comienzo de la consideración de esta disciplina como una especialidad médica científica autónoma ${ }^{1}$. Creador de revistas pioneras en la especialidad ${ }^{2}$, artífice del Primer

1 GRACIA, D. (1971), "Medio siglo de psiquiatría española: 1885-1936", Cuadernos de Historia de la Medicina Española, 10, 305-339; p. 314. Para conocer algunos datos sobre la vida y obra de Giné, se puede consultar, entre otros, el trabajo de REY GonZALEZ, A.M., (1982), "Clásicos de la Psiquiatría Española del siglo XIX. Juan Giné y Partagás (1836-1903)”, Rev. de la Asociación Española de Neuropsiquiatría, II, n 4, 99-110.

2 En concreto fundó, en 1881, y dirigió la Revista Frenopática Barcelonesa que, a pesar de su corta existencia, fue la publicación española de caracter periódico de mayor importancia en su tema durante el siglo XIX. Para más detalle puede consultarse el trabajo de CORBELLA, J. y DOMENECH, E. (1965)., "La 


\section{ANTONIO DIÉGUEZ GÓMEZ}

Certamen Frenopático ${ }^{3}$, fundador de la primera escuela psiquiátrica española y autor del primer tratado, en castellano, que se ocupó específica y exténsamente de las enfermedades mentales ${ }^{4}$, son algunos de los argumentos que respaldan el protagonismo que, sin duda, Giné ejerció en el alienismo español decimonónico. Sobre la obra escrita de este médico catalán ${ }^{5}$, se han vertido algunas opiniones de diferente calado, citándole tangencialmente en ocasiones, repitiendo de manera mecánica observaciones más o menos tópicas sobre la misma en otras, e incluso se ha realizado algún estudio con mayores pretensiones de globalidad que, a nuestro juicio, queda lejos de ofrecer una aportación "definitiva" sobre el tema ${ }^{6}$. Un análisis detallado y profundo, que abarque todos los aspectos del pensamiento psiquiátrico de Giné, es un trabajo ambicioso que consideramos está por hacer.

Nuestro estudio se propone el objetivo de examinar uno de los aspectos de la obra psiquiátrica de Giné, el relativo a su concepción nosográfica, que debido a la consideración que este problema tenía dentro del discurso psiquiátrico de la época en que vivió este autor, no nos debe parecer un asunto menor.

El pensamiento de Giné en relación al tema que nos ocupa puede plantear algunas dificultades para su comprensión. Aunque a todo lo largo de su texto fundamental, el citado Tratado Teórico-práctico de Freno-patología, nos da pistas sobre sus concepciones nosográficas, escuelas y autores que pudieron haber influído en las mismas, e incluso dedica específicamente un capítulo de la obra a tratar esta cuestión ${ }^{7}$, lo cierto es que existen algunas ambivalencias, quizá alguna incongruencia en aspectos conceptuales y epistemológicos que requieren algún detenimiento.

En todo caso, esta etapa de la historia de la medicina en la que Giné desarrolla su labor clínico-teórica, se caracteriza precisamente por la falta de consenso y por las

Revista Frenopática Barcelonesa y el Manicomio de Nueva Belén", en Bases Históricas de la Psiquiatría Catalana Moderna. Barcelona. 1987. pags. 211-217.

3 VIllasANTE, O. (1997), El Primer Certamen Frenopático Español (1883) y su papel en el proceso de institucionalización de la psiquiatría. Tesis doctoral. Universidad Autónoma de Madrid.

${ }^{4}$ GINÉ Y PARTAGÁS, J. (1876), Tratado Teórico-práctico de Freno-patología ó Estudio de las enfermedades mentales fundado en la clínica y en la fisiología de los centros nerviosos. Edit. Moya y Plaza. Madrid.

5 Nos referimos, en todo momento, a la obra psiquiátrica exclusivamente, pues sabido es que Giné ejerció una actividad relevante en otras áreas del saber médico — higiene, dermatología, cirugía, etc-. que en este momento se apartan de nuestro interés. Un somero repaso de estos aspectos puede encontrarse en DOMENECH, E., CORBElla, J. (1969), "Las vertientes principales en la obra médica de Giné y Partagás". Asclepio. vol . 21. p. 173-178.

6 P. ej. el realizado por SANCHO DE SAN ROMAN, R., (1960), La obra psiquiátrica de Giné y Partagás. Tesis doctoral. Editada en el Seminario de Historia de la Medicina. Universidad de Salamanca. (entre las virtudes de este trabajo, no está la de haber realizado un análisis comparativo de suficiente profundidad, respecto de lo que era el saber psiquiátrico en el contexto histórico de Giné)

7 GINÉ Y PARTAGÁS, J. (1876), capítulo XVI, p. 238-245. 
contradicciones especialmente en aquello que define y da nombre a su primer período, al menos en el terreno psiquiátrico, la nosografía.

Porque aunque toda la psiquiatría del siglo XIX es formalmente anatomoclínica y en particular después del "giro copernicano de la lesión anatómica"8, los esfuerzos para ordenar y clasificar los desarreglos mentales, comenzados ya al final de la Ilustración, marcarán la siguiente centuria, siguiendo caminos diversos, encontrados muchas veces, discutida y cuestionada su necesidad y su sentido otras, hasta alcanzar el máximo reconocimiento en la gran sistematización kraepeliniana.

Fueron muy pocos los autores que consiguieron sustraerse a la pretensión de realizar su propia aportación nosográfica e incluso, a veces, nosológica (según el modelo de descripción o "descubrimiento" y ordenación de entidades que se suponen ya preexistentes). No obstante, también aquellos menos tentados por el afán sistematizador -quizá los menos próximos al ámbito francés-, hicieron aportaciones conceptuales en ocasiones decisivas para el debate de fondo. Tal es el caso, por ejemplo, de Guislain o de Griesinger, dos autores que, como veremos, ejercen una influencia importantísima en las concepciones nosográficas de Giné y Partagás. Para la mayoría, sin embargo, se podrá aplicar el comentario que Guislain pone en palabras de Buchez: "así como todo retórico al terminar sus estudios quiere escribir una tragedia, no hay médico mentalista que al llegar al fin de su carrera no quiera hacer una clasificación"9.

De esta manera, el propio Giné, tras examinar una por una todas las propuestas clasificatorias que considera relevantes, de entre las que le preceden, se decide, según sus propias palabras, a "fundar una clasificación, que respondiendo a los designios expresados en las precedentes, al propio tiempo que tienda a proporcionar alguna noción anatomo-patológica, inspire los fundamentos más esenciales de la semiótica y de la terapéutica"10. Tomada literalmente, esta declaración de principios que hace el autor para su clasificación, indica en primer lugar que la manifiesta adscripción a los postulados positivistas y somaticistas de Giné, no le impide llegado el caso adoptar posiciones teóricas eclécticas y/o actitudes científico-clínicas con mayor fundamento pragmático.

\section{LA VOCACIÓN PRAGMÁTICA DE GINÉ}

El mismo espíritu utilitarista y práctico que guia toda la obra de Giné, se puede decir que rige para su esquema nosográfico. Así reza al comienzo de su capítulo

\footnotetext{
8 Expresión que Laín utilizó para señalar el momento en que la lesión deja de estar subordinada al síntoma y pasa a convertirse en el centro del saber patológico y del quehacer clínico. (GRACIA, D., 1971, p. 310)

9 GuISLAIN, J. (1852), Leçons orales sur les phrénopathies. Para este trabajo hemos utilizado una traducción al castellano de M. Carreras Sanchís y J. Torres Fabregat, titulada Lecciones orales sobre las Frenopatias ó Tratado Teórico-práctico de las enfermedades mentales. Madrid. 1881. Tomo 1. p. 69.

10 GinÉ Y PARTAGÁs, J. (1876), p. 240.
} 
dedicado al tema: "El objeto fundamental de una buena clasificación clínica ha de ser proporcionar el mayor número posible de utilidades prácticas"11. Esta orientación eminentemente práctica, buscando la eficacia terapéutica por encima de consideraciones teóricas o doctrinales ha sido señalada por R. Huertas como una de las características y aportaciones de todo el alienismo español decimonónico ${ }^{12}$.

Cuando a juicio de Giné, el problema a tratar requiere una actitud práctica y ponderada, tiende a relativizar sus principios teóricos, se muestra conciliador en las propuestas terapéuticas, e incluso llega, en lo relativo a la clasificación, a renunciar expresamente por este mismo motivo al criterio localizacionista como eje director en el momento de su diseño: "a pesar de las marcadas aficiones anatómicas que hemos manifestado en el decurso de esta obra, renunciamos a todo conato de clasificación basada en el sitio de las lesiones que producen los trastornos funcionales, porque, hoy por hoy, esta idea sería más especulativa que práctica, en razón a que no tenemos suficientes datos para establecer estas localizaciones, y aún cuando los tuviéramos, ni sabríamos ponderar con precisión el valor clínico de estas lesiones, ni tendríamos a nuestra mano una terapéutica pronta a obrar según el sentido de las indicaciones sugeridas por el sitio y naturaleza del daño encefálico"13.

En el mismo sentido se expresa J. Guislain, en nuestra opinión su más claro inspirador en materia freno-patológica general y en cuanto a la esencia de su concepción nosográfica en particular: "una clasificación fundada únicamente en la anatomía patológica, como las propuestas por Parchappe y Voisin, es todavía imposible en el momento actual" 14 . Esto no significa, naturalmente, que para un autor como Giné, sin duda alguna defensor del paradigma anatomo-clínico, con plena vigencia en ese momento, a pesar de su indemostrabilidad (salvo para la parálisis general que provocó su alumbramiento), la alusión al "órgano dañado" fuese una necesidad renunciable. Así podemos ver, por ejemplo, como conoce el planteamiento clasificatorio de A. Voisin, recien aparecido ${ }^{15}$, y no deja pasar la oportunidad para ensalzarlo por estar basado fundamentalmente, aunque no de manera exclusiva, en la anatomía patológica ${ }^{16}$.

11 Ibid. p. 238.

12 HUERTAS, R.(1995), "La psiquiatría española del siglo XIX. Primeros intentos de institucionalización". en Un siglo de Psiquiatría en España. Extra Edit. Colección Salud Mental: Pensamiento y Práctica. Madrid. 21-40. pg. 32. Esta misma tendencia, decididamente pragmática, referida a la nosografía, es subrayada por el mismo autor en la obra esquiroliana: HUERTAS, R. (1997). "Nosografía y antinosografía en la psiquiatría del siglo XIX: a propósito de la psicosis única". RAEN. (en prensa).

13 GinÉ Y PARTAGÁs, J. (1876). p. 238

14 GUISLAIN, J. (1852), p. 71.

15 GiNÉ Y PARTAGÁs, J. (1876), p. 238-239. (aunque la referencia que da Giné resulta algo confusa, la obra donde aparece la clasificación comentada se trata, con toda probabilidad, de las Leçons cliniques sur les maladies mentales et sur les maladies nerveuses, de Augusto-Félix Voisin, publicada en París en 1876, el mismo año que apareció el tratado de Giné.)

16 Ibid. p. 239. 
Giné no sitúa el problema en un nivel epistemológico y tampoco parece dudar sobre la metodología a seguir para la aprehensión y ordenamiento nosotáxico de las enfermedades mentales. Piensa que se trata de perseverar en la búsqueda, que es un problema de tiempo, del porvenir científico, puesto que sería el limitado "estado actual de la ciencia" el que condicionaría el grado de conocimiento alcanzado. "Lícito es, sin embargo, confiar en que no está lejano de nosotros el día en que conociendo detalladamente ese funcionamiento específico de cada una de las partes que constituyen este órgano múltiple llamado cerebro, vistas las anomalías que ofrecen estas mismas funciones, nos será dable colegir la lesión material que las determina y por consiguiente, podremos caminar desembarazadamente por la vía del diagnóstico anatómico"17. Pero mientras estos avances no se producen el esfuerzo de adaptación a los parámetros de la realidad - de los cauces del conocimiento clínico del momento- que Giné realiza al plantear su sistema nosográfico, le conduce a adoptar unos criterios nosotáxicos de referencia que podríamos afirmar tienen mayor fundamento "psicologista" que "somaticista". Esto es así si tenemos en cuenta que cuando Giné hace una llamada a la práctica, desemboca casi constantemente en Guislain, que, no solo representa en el terreno nosográfico las concepciones más "psicologistas", sino que incluso podríamos decir que su obra se sitúa en los aledaños de la antinosografía.

Cuando Guislain habla de las "seis manifestaciones fisiológicas" del comportamiento o del psiquismo humano, sobre cuyo patrón ajusta posteriormente las seis "formas elementales" de sus frenopatías ${ }^{18}$, está refiriéndose, sin más, a conductas o síntomas, en cualquier caso manifestaciones de naturaleza psicológica. También es el mismo Giné, que aparece como abanderado del organicismo, quien se expresa diciendo: "reduzcamos pues, modestamente nuestras aspiraciones nosotáxicas al perímetro de la Fisiología, ya que lo único que conocemos positivamente de los enfermos mentales es su sintomatología, ó sean los trastornos funcionales"19. Pero, como ya señaló con acierto Sancho de San Roman ${ }^{20}$, esa "fisiología", es en Giné psicología. Tampoco ha de resultarnos extraño si acudimos a Griesinger, otro referente clave en la nosografía de Giné, y reparamos en que el célebre autor de la archiconocida frase "las enfermedades mentales son enfermedades del cerebro" - todo un eslogan organicista que no resume de manera acertada la complejidad de su obra- es, a la vez, quien afirma que "en lugar de un principio de clasificación anatómica, debemos tomar como base las funciones, la fisiología y, como en la alienación los cambios

\footnotetext{
${ }^{17}$ Ibid.p. 213

18 GUISLAIN, J. (1852), p. 72-73.

19 GINÉ Y PARTAGÁs, J. (1876). p.239.

${ }^{20}$ SANCHO DE SAN ROMAN, R. (1960), p. 16.
} 
señalados en la inteligencia y en los sentimientos afectivos son las lesiones capitales y las más llamativas, la psicología"21.

Este trasfondo "psicologista" al que irían anclados, en virtud de una finalidad práctica, los planteamientos nosográficos de Giné, resulta en última instancia muy compatible con las consecuencias terapéuticas que se derivarían del mismo. Dicho de otra manera, si para la mayor parte de las entidades clínicas que se incluyen en la clasificación se planteara la imposibilidad terapéutica - como ocurriría si se basara su diagnóstico y su existencia en una misteriosa e inabordable localización anatómica-, la especialidad médica que tuvo a Giné como principal artífice de su introducción en España, quedaría definida como terapéuticamente estéril. Como sabemos, ocurre más bien al contrario, es a expensas de una -en muchas ocasiones- amplia oferta terapéutica, como se organiza y legitima el desarrollo teórico y sobre todo asistencial de la especialidad $^{22}$. No cabe duda que parte fundamental de esa oferta terapéutica es el llamado tratamiento moral, ya criticado pero todavía imperante en esta etapa, con el aislamiento asilar como elemento central del mismo, que ganaba tanto más sentido cuanto más susceptibles de tratar "moralmente" eran los trastornos mentales definidos. A éste respecto la actuación de Giné estuvo lejos de disentir de lo que "en España fue asumido tanto por idealistas como por materialistas"23. "La primera indicación que se presenta, al emprender el tratamiento de la alienación mental, consiste en la sustracción a las influencias físicas y morales que han preparado y aún ocasionado la enfermedad. El medio indicado es, en este caso, el aislamiento"24.

${ }^{21}$ GrIESINGER, W. (1861), Die Pathologie und therapie der psychischen Krankheiten. $2^{\mathrm{a}}$ edic. Para este trabajo hemos consultado la traducción francesa de Doumic, titulada Traité des Maladies mentales. Pathologie et therapeutique. París. 1865. p. 246.

22 En este sentido Giné manifiesta un "optimismo terapéutico" absolutamente coherente con los postulados del empirismo psiquíatrico que, anclando su raíz en la Ilustración, definirá a continuación la clínica psiquiátrica del siglo XIX.

23 HUERTAS, R. (1995). p. 25. La introducción del tratamiento moral en España, que tiene en la labor de Pí y Molist el máximo protagonismo (véase su Proyecto médico razonado para la construcción del Manicomio de la Santa Cruz, -1860-) ha sido bien estudiada por COMELLES, J.M.,(1988), La razón y la sinrazón. Asistencia psiquiátrica y desarrollo del estado en la España contemporánea. PPU. Barcelona. pags. 53-76., y por PESET, J.L., (1993), "Entre el gabinete y el manicomio: reflexiones sobre la psiquiatría española de fin de siglo", en GONZALEZ DE PABLO, A. (coord.), Enfermedad, clínica y patología. Estudios sobre el origen y desarrollo de la medicina contemporánea. Madrid. Edit. Complutense, pags. 281-299.

24 GINÉ Y PARTAGÁs, (1876). p. 298. No hay que olvidar que Giné era director de uno de estos lugares de aislamiento terapéutico, el manicomio de Nueva Belén, aunque se aparta del objetivo de este trabajo el análisis de las implicaciones que este hecho pudiera suponer para las cuestiones planteadas. Cabe pensar, no obstante, que ninguna propuesta de clasificación de los trastornos mentales se podría decir que es ateórica o ideológicamente ingénua (probablemente no se podría afirmar ésto de ninguna clasificación en general). La suscripción de un modelo taxonómico u otro conlleva, normalmente, la adscripción a una teoría conceptualizadora de los fenómenos, objetos, etc, que se propone clasificar, y a una ideología no necesariamente reconocida. Más aún, pensamos que la personalidad del autor o sus- 


\title{
ECLÉCTICO EN SU PROPUESTA NOSOGRÁFICA
}

Si atendemos exclusivamente a la presentación que de sus concepciones teóricas hace Giné a lo largo de toda su obra, en la que queda autodefinido como auténtico paladín de las teorías positivistas, el planteamiento del epígrafe carecería de sentido. Sin embargo, en consecuencia con todo lo dicho anteriormente, resultaría coherente atisbar en el pensamiento nosográfico de Giné, un planteamiento ecléctico e integrador. La afirmación, no obstante, deviene contradictoria cuando es el mismo autor quien nos da argumentos para sostenerla por un lado y repudia taxativamente esa posibilidad por el otro.

En efecto, en sus Lecciones sobre Historia de la Medicina, dadas durante el curso de 1868-1869, para los alumnos del doctorado de la Facultad de Medicina de Barcelona, Giné hace la siguiente advertencia:

\begin{abstract}
“ $\{$ Del eclecticismo $\} ..$. \{De qué modo el eclecticismo es la negación de todos los sistemas y una rémora para el progreso $\}. . . . E l$ eclecticismo no exije, pues, que se hagan nuevos descubrimientos, ni que se inventen hipótesis más o menos ingeniosas, sino que se proceda a un examen más o menos riguroso de lo que otros hicieron y de lo que otros descubrieron, para aprovecharse de lo que en la experiencia y en la ciencia agena hay de útil y verdadero....Los eclécticos se erigen árbitros en medio de las discusiones de los demás sistemas y atienden a todos....Preguntad, sino, á un ecléctico, como distinguirá lo verdadero de lo falso, lo útil de lo pernicioso de las partes de un sistema, y os contestará que para ésto está el buen sentido, o el criterio individual. Es decir, pues, que el eclecticismo es el individualismo racional. Pero un individuo no puede formar una escuela ¿cómo, pues, tendrá razón de ser la escuela ecléctica con su individualismo?.... Los eclécticos no quieren reñir con nadie y no tienen ningún amigo; son en medicina como esos políticos acomodaticios, que vuelven el rostro del lado del sol que más calienta; son y no son a la vez; si algo son, son la negación de toda idea. Y es preciso hablar así del eclecticismo, porque conviene a toda costa presentar con toda su ridícula desnudez a un pseudo-sistema, que mata todas las aspiraciones del progreso. Protestando el ecléctico de que él no debe hacer más que escojer, que su condición especial no le obliga a descubrir nada nuevo, eleva al rango de virtud científica la holganza y es sirena engañadora que adormece el ardor para el trabajo que siente la juventud."25
\end{abstract}

El tono adoptado por el autor de este texto, siete años después, cuando escribe sobre la clasificación de las enfermedades mentales, es bien distinto. Sus puntos de vista son más contenidos y los acercamientos a las distintas teorías y posiciones de otros autores son realizados en un talante mucho más integrador.

La multiplicidad de influencias que, aunque con desigual repercusión, se pueden apreciar en la sistemática de Giné, es fundamentalmente lo que permite sostener esta

criptor de cualquier sistema taxonómico e incluso determinados intereses extracientíficos del mismo, generalmente no explicitados, pueden jugar un papel relevante en la elección de ese sistema clasificatorio.

${ }^{25}$ GINÉ Y PARTAGÁs, J. (1869). Lecciones sobre Historia de la Medicina. Barcelona. p. 168-169. 


\section{ANTONIO DIÉGUEZ GÓMEZ}

afirmación. Cuando aborda el estudio de cualquier problema, muestra en todo momento una tendencia a hacerlo de manera enciclopédica, demostrando un conocimiento suficiente, cuando menos, de casi todos los autores importantes de su época y del pensamiento, al respecto, de sus predecesores. Un buen ejemplo de ésto lo constituye, precisamente, la exposición de su propuesta nosográfica. Cuando entra en esta materia, lo hace realizando un recorrido por los esquemas clasificatorios de casi todos los autores que considera importantes ${ }^{26}$, poniendo de manifiesto un notable grado de familiarización con las concepciones nosográficas de los mismos. Analiza con algún detalle las ventajas y los "defectos" que a su parecer plantean cada una de estas propuestas, con la manifiesta intención de "abarcar cuanto hay de útil en los diferentes ensayos de clasificación de las enfermedades mentales y vengamos a establecer la que nos sirve de pauta en nuestra clínica"27.

Después de examinar detenidamente cada uno de los apartados que Giné establece en su clasificación, quedan de relieve, a nuestro juicio, dos aspectos cruciales para su comprensión. Por un lado la multiprocedencia de las distintas categorías y agrupaciones diagnósticas. En segundo lugar un elemento de cohesión, al que el autor se refiere constantemente, buscando lo que podríamos llamar una "idea de conjunto".

En cuanto a la primera cuestión, podemos decir que la clasificación de Giné resulta de un ensamblaje de categorías y agrupaciones nosológicas, tomadas de distintos autores y ocasionalmente matizadas. Aunque la procedencia geográfica y el ámbito teórico y científico de los mismos es variado, el peso que determinadas fuentes en principio de procedencia no francesa, tienen en las ideas nosográficas de Giné, permite cuestionar seriamente la "clara dependencia del pensamiento francés" 28 en lo relativo, al menos, a su pensamiento nosográfico. Más adelante podremos ver con mayor detenimiento, como, en efecto, es cuantitativa y cualitativamente arrolladora la presencia de planteamientos nosográficos concretos de Guislain y de Griesinger en la obra del psiquiatra catalán ${ }^{29}$. También ocupan un lugar otros autores pertenecien-

\footnotetext{
${ }^{26}$ Resulta llamativa la omisión, en esta exposición preliminar, de la clasificación de Griesinger, sin duda un autor de los que más aspectos recoge Giné, en materia de clasificación.

27 GINÉ Y PARTAGÁS, J. (1876), p. 238.

${ }^{28}$ Afirmaciones como ésta (GRACIA, D. -1971-, p. 314), resultan, sin embargo muy coherentes con la idea unánimemente aceptada sobre el caracter decisivo que, para la medicina general y otros ámbitos de la ciencia y de la cultura, tuvo la influencia francesa en esa etapa de la historia. (vease p. ej. para la medicina, LOPEZ PIÑERO, J.M.-1964- Medicina y Sociedad en la España del Siglo XIX. Sociedad de Estudios y Publicaciones. Madrid. p. 85, donde se expresa esta idea en toda su radicalidad).

29 PESET, V. (1954), en el apéndice a su traducción de La Historia de la Psicología y de la Psiquiatría en España desde los tiempos más remotos hasta la actualidad, de ULLERSPERGER, J.B. (1871), Edit. Alhambra. Madrid. (1954), p. 193, también defiende la idea de que en España continúa imperando hasta fin de siglo la psiquiatría francesa. La afirmación, referida al panorama global de la psiquiatría española de la época, es poco discutible. Tomando como referencia la obra de Giné, no podemos compartir, en cambio, el resto del contenido de la misma, en el que asegura que el Lehrbuch de Griesinger tuvo poca repercusión en nuestra patria y aún más "salvo los del anatomopatólogo Rindfleisch y el de Meynert, los
} 
tes al ámbito propiamente francés, como Esquirol, Baillarger, etc, que a pesar de ejercer una influencia directa de mucho menor alcance que la de los anteriores, permiten abonar la hipótesis del eclecticismo nosográfico en Giné. Si bien no podemos decir en absoluto que aquellos - Guislain y Griesinger - fueron autores ajenos a los círculos franceses ${ }^{30}$, en lo relativo a sus ideas nosográficas no representan el "sentir" de la psiquiatría francesa del momento. Más bien, se podría decir lo contrario, que tanto el "Pinel belga" como el psiquiatra alemán, figura clave de la psiquiatría decimonónica de ese país, reconocidos "unitaristas", podrían estar próximos a la psiquiatría antinosográfica.

Antes de desarrollar pormenorizadamente estas ideas, señalar el segundo aspecto al que antes me refería como característico del sistema de clasificación de Giné, con el que el autor trasmite una "idea de conjunto" en su ordenamiento nosotáxico. Se trata de la aplicación sistemática, en primera instancia a veces, o después de haber realizado un planteamiento more botanico ortodoxo para una determinada agrupación nosológica en otras, del "concepto nosológico general" guislainiano, como llama Giné al modo en el que en todo caso se deben clasificar las enfermedades mentales: formas simples y formas compuestas.

\section{"ESPECIES MORBOSAS" VERSUS "FORMAS O VARIEDADES". ALCANCE DE LOS POSTU- LADOS UNITARISTAS EN GINÉ.}

El hecho de que la obra psiquiátrica de Giné esté, al menos en un principio, inspirada de manera fundamental en los autores antes referidos, no parece una cuestión intrascendente ${ }^{31}$. Sin duda no lo es si atendemos a sus resultados, ya que el sesgo que la influencia de estas fuentes imprime a su obra pone en entredicho el voceado positivismo que caracterizaría los entresijos de la misma. Cuando aparece el texto psiquiátrico principal de Giné, el interminable debate sobre el problema de las nosografías que

\footnotetext{
nombres que vemos en el libro de Giné siguen siendo franceses...". A lo largo del texto daremos cuenta de la notable presencia que la obra de Griesinger tiene en el pensamiento nosográfico de Giné.

30 Es conocida la influencia y consideración que el célebre tratado de Griesinger, Die Pathologie und Therapie der psychischen Krankheiten, tuvo entre los alienistas franceses. No en vano es a través de su traducción al francés de 1865, anteriormente citada, como, fundamentalmente, fue difundido entre nosotros y como más asequible nos sigue resultando.

31 Desconocemos las motivaciones íntimas que pudieron llevar a Giné a realizar esta elección. El análisis de las mismas podría ser objeto de un interesante estudio. Sabemos que el gran alienista francés Brièrre de Boismont, muy considerado por entonces en España, dirá en 1866, sobre las Leçons orales de Guislain, que “...es el tratado más práctico de los tiempos modernos..” (citado por BERCHERIE, P. (1980), Les Fondements de la Clinique. Histoire et structure du savoir psychiatrique. Edit. Navarín. París. Se ha utilizado la traducción al español de edit. Manantial. Los fundamentos de la clínica. p. 47.). Por alguna razón Giné pensaba de igual manera.
} 


\section{ANTONIO DIÉGUEZ GÓMEZ}

atraviesa todo el siglo XIX, se encuentra bastante avanzado. La mayor parte de las figuras del alienismo decimonónico han publicado sus obras y expresado sus puntos de vista al respecto. Han pasado más de tres lustros desde el intenso debate celebrado sobre el tema en el seno de la Société Médico-psychologique ${ }^{32}$, y los distintos autores europeos han definido ya sus posiciones teóricas sobre el problema, aunque como veremos, no siempre podrán mantenerlas dentro de una dialéctica coherente.

Como ya hemos advertido, Giné demuestra un cumplido conocimiento del estado de la cuestión, y en estas condiciones fundamenta la estructura de su nosografía -y por extensión de su saber psiquiátrico- sobre la obra de un alienista francófono, pero de origen y radicación belga. En efecto, son las Leçons orales sur les phrenopathies, de Joseph Guislain, publicadas en 1852, el texto que más influye en el pensamiento nosográfico de Giné, seguido en importancia por la obra de Griesinger, que, además, es el autor que se encargará de recoger y dar el máximo auge a las ideas que sobre la entidad y clasificación de las enfermedades mentales tenía el médico de Gante. En estos dos autores pueden encontrarse claramente las líneas básicas que sobre la clasificación de las "frenopatías" 33 hereda Giné. La relevancia del papel jugado por la obra de ambos en la génesis y desarrollo de la teoría de la Psicosis Unitaria es de sobra conocida. Las posiciones teóricas con respecto a la posibilidad o conveniencia de clasificar los desarreglos mentales, adoptadas por quienes a lo largo de la historia de la psiquiatría han defendido - no siempre de manera explícita - las tesis unitaristas, han oscilado entre el rechazo absoluto, negando cualquier sentido a la clasificación (Jacobi, H. Neumann) ${ }^{34}$ y la aceptación de "formas" o "variedades", dentro de un mismo proceso, que permitirían explicar las diferencias entre unos casos y otros sin suscribir un principio natural en la división (Griesinger) ${ }^{35}$. Pero antes de analizar con mayor detalle la repercusión que sobre la nosología y nosografía de Giné ejercen dos de los autores más representativos de la Einheitspsychose $e^{36}$, se hace necesario, para una adecuada contextualización, un mínimo rastreo históricoconceptual del problema ${ }^{37}$.

${ }^{32}$ El texto íntegro de las sesiones puede encontrarse en Annales Médico-psychologiques, $3^{\text {a }}$ serie, tomo VII, 1861.

33 También en terminología la impronta guislainiana en la obra de Giné es determinante.

${ }^{34}$ KAHLBAUM, L. (1863) Clasificación de las enfermedades mentales. Colección Clásicos de la psiquiatría. DOR. S.L. Madrid, 1995. p.90. .

35 Berrios, G., BeEr, D., (1995) “Unitary Psychosis Concept”. En Berrios, G., PORTER, R., A History of Clinical Psychiatry. The Athlone Press. Londres. 313-335. p.313.

36 Según COLOdRón, A. (1990), Las Esquizofrenias. Sindrome de Kraepelin-Bleuler. Edit. siglo XXI. $2^{a}$ edic., Madrid. p. 29., ésta es la expresión primitiva en aleman, para la psicosis unitaria, que comienza a divulgarse con Griesinger.

37 Aunque en la etapa cronológica en que centramos nuestra atención, la teoría de la P. Unitaria ya había iniciado su declive, con posterioridad experimenta períodos de reactivación, en particular hacia mediados del presente siglo, debido a obras tan importantes como las de H. Ey, B. Llopis, W. Janzarik, 
Son múltiples los trabajos de autores solventes que, refiriéndose a los primeros alienistas importantes del siglo XIX, encuentran ya en Pinel el primer referente de lo que puede ser una concepción unitaria de la enfermedad mental ${ }^{38}$. Podría resultar paradójico pensar que, precisamente el autor de la Nosographie Philosophique, sea a la vez el más conspicuo representante de una época en la que, como dice P. Pichot, "se utilizaba únicamente el término alienación para designar los trastornos mentales", entendiendo que "la alienación mental era una enfermedad susceptible de manifestarse bajo aspectos sintomáticos diferentes, pero la consideraban como fundamentalmente única en su naturaleza" ${ }^{39}$. Esta aparente incongruencia - toda una obra dedicada a clasificar un único trastorno-, se va desvaneciendo al razonar con Guiraud que "los nosógrafos de este período describen más bien diversas variedades de la locura o de la alienación mental que enfermedades verdaderas en el sentido de la patología general. Los enfermos pasan fácilmente de una enfermedad a otra (....) es decir, no se trata más que de agrupaciones de síntomas correspondientes a un momento determinado y no a la evolución entera de la enfermedad" 40 . Exactamente en el mismo sentido se expresan Berrios y Beer ${ }^{41}$ y, con mayor contundencia aún, Morales Meseguer al asegurar que "en esta etapa la psiquiatría francesa no se había planteado aún de forma explícita el problema de la existencia de especies morbosas distintas en el área de la patología mental"42. Según todo ésto, no debería resultarnos extraño que Pinel utilizara el singular en el título de su otro gran texto, el Tratado Médico-filosófico de la alienación mental o Manía.

Más allá de la trascendencia de alguna de sus aportaciones nosológicas ${ }^{43}$, se ha querido ver en la nosografía esquiroliana un señalado precedente de la concepción

\footnotetext{
etc. Nosotros limitaremos nuestro recorrido exclusivamente al período de la pasada centuria que antecede a Giné. De entre los distintos trabajos que abordan la historiografía de este problema, resulta más completo, por abarcar todas las épocas, el ya mencionado de BERRIOS, G. y BEER, D. (1995).

38 Como ejemplos citaremos los de Guiraud, P., (1950), "Evolution de la nosographie clinique", en su Psychiatrie Générale. Edit. Le François. París. p. 17-34. p.20.; PICHOT, P. (1997), "El concepto de cronicidad", en Cronicidad en Psiquiatría. Gutierrez Fraile, M., Ezcurra, J., Pichot, P. (edits). Ediciones en Neurociencias. Barcelona . p. 21-22.; BERRIOS, G., BEER, D. (1995).; HUERTAS, R. (1997), "Nosografía y antinosografía en la psiquiatría del siglo XIX: a propósito de la psicosis única" en RAEN. (en prensa). No nos detendremos en la alusión que hacen algunos autores, -p. ej. LLOPIS, B., (1954), "La Psicosis Unica", Archivos de Neurobiología. XVII. 1. 3-41. p. 6-7.- a Areteo de Capadocia, como primer indicio de esta teoría. Aunque sabemos que Giné conocía sobradamente este precedente histórico - p. ej. se refiere a él como "el gran nosógrafo de la antigüedad" en la pag. 4 de su libro-, parece poco probable una influencia directa del citadísimo clásico, contemporáneo de Galeno, dada la gran separación en el tiempo.

39 PICHOT, P. (1997), p. 21.

40 GuIRAUD, P. (1950) 17-34. p. 20

41 BERRIOS, G. BEER, D. (1995), 313-335. p. 17.

42 MORAles MeSEguer, J.M. (1974), "Psiquiatría", en LAIN, P. (dir.). Historia Universal de la Medicina. tomo VI. Edit. Salvat. 217-228, p. 219.

43 Determinante, como se sabe, la de la Monomanía, por su importantísima repercusión jurídico-social.
} 
unitaria de la enfermedad mental ${ }^{44}$. Así, para las agrupaciones de Esquirol se podría decir lo mismo que anteriormente señalamos para las especies pinelianas: que no serían sino formas o variedades de un único proceso. Al referirse a los síntomas de la locura, alude a las especies de Pinel como "formas que puede adoptar la locura sucesiva y alternativamente" 45 . No obstante, esta fidelidad nosográfica al maestro, compartiendo la misma idea sobre la unidad genérica de la locura ${ }^{46}$, no es entendida así por todos los autores ${ }^{47}$. En nuestra opinión, quizá no debiera buscarse, en la nosografía de Esquirol, un rigor y una coherencia que probablemente no se propuso el propio autor.

Para otros renombrados alienistas de la época, como es el caso de E. J. Georget, no se advierten importantes disensiones con respecto a sus maestros en esta materia $^{48}$. Incluso a pesar de la consabida reivindicación de la estupidez como un nuevo "género" sobre la demencia aguda de Esquirol ${ }^{49}$, para Llopis, el precozmente desaparecido discípulo de éste "desarrolló con mayor precisión el concepto de la unidad de todas las psicosis en su libro De la folie" 50 .

Es casi un lugar común la afirmación que sitúa el origen manifiesto de la teoría unitarista de la locura en la influencia que la obra de J. Guislain ejerce sobre E.A. Zeller ${ }^{51}$. Para Guislain hay un estado de "dolor moral" o "frenalgia" que se encuentra en la base de todos los trastornos anímicos ${ }^{52}$. Posteriormente la combinación y transformación de una enfermedad en otra darían como resultado cualquiera de las "formas" que caracterizan la nosografía de este autor y para las que Zeller establece una secuencia más definida: melancolía-manía-paranoia-demencia, que representarían "formas clínicas" de un proceso único. La posibilidad de la curación alcanzaría hasta

\footnotetext{
44 BERrIOS, G., BEER, D., (1995), p. 317.

${ }^{45}$ ESQUIROL, J.E.D., (1838), Des maladies mentales considérées sous les rapports médical, hygiénique et médico-legal. Hemos utilizado la traducción al castellano de R. de Monasterio y Correa, titulada Tratado completo de las Enagenaciones Mentales consideradas bajo su aspecto médico, higiénico y médico-legal. Imp. del Colegio de sordomudos. Madrid. 1847. Tomo I. p. 10.

46 ALVAREZ, J.M. (1994), "Una historia del delirio en la clínica francesa", en El delirio en la clínica francesa. Colección clásicos de la psiquiatría. Edic. Dorsa. Madrid. p. 31.

${ }^{47}$ HUERTAS, R., (1997). (e.p.). En lo que verdaderamente está interesado Esquirol es en establecer diagnósticos diferenciales claros, no tanto, pensamos nosotros, en ofrecer un ordenamiento nosográfico de los mismos. No demuestra gran interés en aplicar con propiedad la nomenclatura de los distintos niveles de abstracción nosográfica (POSTEL, J., QUETEL, CL., p. 155) y para autores como KAHLBAUM (-1863-, p.53) no habría que conceder mayor valor teórico - sino solamente práctico- a la relación entre las clases que Esquirol establece en su sistema.

48 BERRIOS, G., BEER. D., (1995). p. 322.

49 BERCHERIE, P. (1980), p. 34.

${ }^{50}$ LLOPIS, B. (1954), p. 7.

51 DÖRNER, KL. (1974), Ciudadanos y locos. Historia social de la psiquiatría. Madrid. p. 217 y 364. JACKSON, S.W. (1986). Historia de la melancolía y la depresión. Desde los tiempos hipocráticos a la época moderna.. Edic. Turner. Madrid. 1989. p. 168 y 249.; (También VLIEGEN, J.(1980). Die Einheitspsychose . Stuttgart. Enke. p. 9. citado por Berrios en A History of Clinical Psychiatry)

52 BERCHERIE, P. (1980), p.41.; KAHLBAUM, L. (1863), p. 108.
} 
las dos primeras, quedando la paranoia y la demencia como productos secundarios e incurables ${ }^{53}$. Lo que en Zeller alcanza ya la consistencia de un modelo teórico claramente explicitado, en Guislain aparece con mayor ambigüedad, menor definición, como un esbozo doctrinal, que solo será a través del empuje de Griesinger como se convertirá en una teoría con manifiesto predicamento en el período central del siglo. Ciertamente existe una concepción unitaria en la obra de Guislain, pero en nuestra opinión tendría más bien un caracter implícito, en definitiva no muy diferente del que hemos señalado para los primeros clásicos franceses. La división que hace entre formas elementales y formas compuestas resulta fundamental para entender su nosografía, pues aunque llega a proponer seis "grupos" (refiriendose a las formas elementales) ${ }^{54}$ - basadas en lo que él entiende como las seis manifestaciones fisiológicas básicas del psiquísmo humano- acaba resolviendo en última instancia el problema de la multiplicidad de "caras" de la enfermedad mental, apelando al gran predominio de las formas compuestas sobre las simples o elementales ${ }^{55}$.

Es la gran importancia y prestigio de W. Griesinger, lo que probablemente explica que se haya convertido en el principal referente histórico sobre la teoría de la psicosis unitaria ${ }^{56}$. Esto no significa que los planteamientos más radicales con respecto a esta doctrina correspondieran al recordado neuropsiquiatra aleman. El mayor entusiasmo en la defensa de estas tesis lo puso $\mathrm{H}$. Neumann, quien abogaba, con gran énfasis, por el principio de la metamorfosis de las distintas formas, que - una vez más- no constituirían sino estados de un solo proceso patológico ${ }^{57}$ y que propugnaba de forma tajante la abolición de las clasificaciones: "no podemos creer en un progreso real de la psiquiatría hasta que se haya tomado la decisión general de arrojar por la borda todas las clasificaciones" 58 .

En su obra de mayor divulgación ${ }^{59}$ Griesinger afirma que, pese a su convencimiento acerca del sustrato anatomo-patológico cerebral de las enfermedades mentales, el ordenamiento nosográfico de esta clase, reposa simplemente sobre la sinto-

\footnotetext{
53 Morales Meseguer, J.M. (1974), p. 226.

54 Guislain, J. (1852), p. 72-73. Las seis formas elementales que propone son las siguientes: 1. Melancolía (Frenalgia); 2. Extasis.; 3. Manía.; 4. Locura; 5. Delirio.; 6. Demencia.

55 Ibid. p. 70.

56 El papel de Griesinger con respecto a la teoría de la P. Unitaria, no fue decisivo solo por haber sido el máximo difusor de la misma, sino que también contribuyó de manera determinante a su declive: los trabajos de Hoffmann y Snell que rompen con el dogma de la naturaleza exclusivamente secundaria de la paranoia, fueron claves en este sentido, y no hubieran tenido mucha trascendencia si el propio Griesinger, en 1867, no los hubiese recogido, aceptando la existencia de una "paranoia primaria". (LLOPIS, B. -1954-, p.12.).

57 KahlBaUM, L. (1863), p. 103.

58 LLOPIS, B. (1954), p. 9-10.

59 Aunque Die Pathologie und Therapie der psychischen Krankheiten fue escrito en su juventud (Griesinger aún no contaba treinta años de edad) no alcanzó el prestigio que se le reconoce hasta pasado un cuarto de siglo después. (POSTEL, J. QUETEL, CL. -1987- p.660)
} 


\section{ANTONIO DIÉGUEZ GÓMEZ}

matología, no pudiendose considerar como diferentes géneros de la misma lo que no son más que síntomas diferentes o distintas "formas de locura". Refiere dos grandes grupos de anomalías psíquicas, con la alteración de las emociones como principal eje responsable del primero y las lesiones de la inteligencia y la voluntad en el segundo grupo. En la inmensa mayoría de casos los estados del primer grupo preceden a los del segundo y, si se alcanzan éstos, la enfermedad cerebral no es curable ${ }^{60}$. Como podemos ver, hasta aquí las modificaciones con respecto a su maestro Zeller, pasan desapercibidas. Se hacen notar, sin embargo, cuando Griesinger introduce en el primer grupo mencionado "las formas de la melancolía, manía y monomanía exaltada" y en el segundo "las locuras sistematizadas, demencia parcial y demencia general"61.

Tenemos ya elementos suficientes para entender cuál pudo ser el pensamiento de Giné con respecto a la existencia de un único proceso o bien de múltiples e independientes entidades clínicas. Una primera pista, evidente, nos la da el título de su tratado, que al igual que los de Guislain y Griesinger, habla en plural de "las enfermedades mentales". Giné, clasifica, propone una nosografía y, como los anteriores, lo hace atendiendo a la sintomatología. Si evitamos la conclusión de que los unitaristas son necesariamente antinosológicos ${ }^{62}$, podríamos decir de Giné exactamente lo mismo que E. Balbo afirma sobre el trabajo de Kahlbaum" ${ }^{63}$, que "su intento de fundar una nueva nosografía psiquiátrica, podemos decir que se mueve entre dos polos, el que se encuentra por un lado centrado en la concepción unitaria de la enfermedad y el contrapuesto representado por una doctrina muy ramificada de la enfermedad" ${ }_{64}$. Por lo mismo, creemos que la ambivalencia es la expresión que mejor resume y define la actitud de Giné hacia este problema. Expresión palmaria y perfecta ilustración gráfica de la misma son los términos que constantemente utiliza para referirse a los cuadros clínicos: "especies o formas". De esta manera atiende, por un lado, a la

60 GRIESINGER, W. (1861), p.246-248.

61 Aunque pocos años después (1864), Griesinger se propuso partir de unos nuevos principios en su clasificación, (según BERRIOS, G., BEER, D. -1995-, p.321.), quizás influenciado por la lectura de Kahlbaum, la obra que con toda probabilidad manejó e influyó en Giné fue la antedicha reedición de 1861, a través de su traducción al francés de 1865.

62 BERRIOS, G., BEER, D. (1995), p.313. El problema de la relación entre nosografía y psicosis única queda bien ilustrado en JANZARIK, W., (1969), "Nosografía y psicosis única". En Schizophrenie und Zyklothimie. Stuttgart. Traducción al castellano de Pi Suñer, S. Esquizofrenia y ciclotimia Edit. Morata. Madrid. -1972-. p.51-61

63 BALBO, E. (1995), "La obra de Karl Ludwig Kahlbaum en la psiquiatría alemana del siglo XIX". En Clasificación de las enfermedades mentales. L. Kahlbaum. Colección Clásicos de la psiquiatría. Edit. DOR. S.L. Madrid. p. 24.

${ }^{64}$ Creemos que ese comentario refleja exactamente la posición de Giné en nosografía, pero naturalmente somos conscientes del desigual papel que uno y otro autor han jugado en la historia de la psiquiatría. Es un hecho conocido la decisiva repercusión que los planteamientos clasificatorios del descriptor de la catatonía, tuvieron sobre la sistemática kraepeliniana. Por otro lado es altamente improbable que Giné hubiera tenido ya conocimiento de la obra del aleman. 
tradición nosográfica more botanico y por el otro, aunque de manera simultánea, pone de manifiesto su adscripción al pensamiento unitarista, utilizando el término que, como referencia sindrómica en unas ocasiones, o evolutiva en otras, caracteriza la nomenclatura de los autores que - bien de manera explícita o bien tácita - suscriben esta doctrina. Recoge el "estado frenopático" prodrómico de Guislain, tanto para la melancolía ${ }^{65}$, como para la manía ${ }^{66}$ y establece los dos grupos que anteriormente vimos en Griesinger, el de "las enfermedades mentales que concuerdan entre sí por un estado emocional morboso..." en primer lugar, y en las que "la emoción patológica, exaltante o deprimente, es lo característico...", y en segundo lugar aquellas otras "formas de enajenación mental (...) en las que resalta una degradación más o menos marcada de las facultades intelectuales" 67 . Al igual que sus inspiradores en esta materia, señala que "lo más frecuente es que se vaya a parar a ellas - las del segundo grupo- después de haber pasado por alguna de las formas del primer grupo", dejando de esta manera sentada su conformidad con el criterio de la metamorfosis, propio de los unitaristas. En la misma línea, reconoce la posibilidad de tratamiento solo para los primeros estados, resultando "la terapéutica siempre impotente (...) cuando se revela la degradación de las facultades mentales"68. Con todo, para la tan aireada mentalidad positivista de Giné, deben parecer demasiadas concesiones a la relatividad de los hechos y, amparándose en imperativos de la práctica clínica, se ve obligado a decir que "la división dicotómica que acabamos de establecer, es más clínica que nosológica; porque en teoría no podría aceptarse una división capital en una clase de enfermedades que pueden las de una género pasar a constituir especies del género antitético"69.

Otro aspecto que hemos considerado característico de la nosología guislainiana, la socorrida distinción entre formas elementales y formas combinadas, es incorporado en los principios clasificatorios de Giné con tal interés que incluso sirve a éste como argumento, entre otros, para rechazar la clasificación de Esquirol, por la "imposibilidad de incluir en ella las formas mixtas, ó compuestas, que con tanta frecuencia se observan en la práctica" 70 . En consecuencia traslada literalmente a su tratado el extenso cuadro sinóptico de las formas compuestas de las frenopatías que aparece en el de Guislain ${ }^{71}$.

65 GINÉ Y PARTAGÁS, J. (1876), p. 391.

66 Ibid.p. 359.

${ }^{67} \mathrm{Ibid}$. pags. 213-214. Aunque en este segundo grupo Giné solo incluye a las demencias, no hay diferencia alguna con respecto a las formas que antes dijimos incluía Griesinger en el mismo grupo, ya que todas ellas se aglutinan en los "estados de decaimiento intelectual" de este último, expresión que se corresponde exactamente con las demencias de Giné.

68 Ibid.p. 214

${ }^{69}$ Ibid. p.214.

70 Ibid.p. 240

71 Ibid. el cuadro aparece inserto entre las p. 214 y 215. 


\section{ANTONIO DIÉGUEZ GÓMEZ}

\section{LAS CATEGORIAS Y SU ORDENAMIENTO.}

Como venimos diciendo, junto a esta relativización de las divisiones nosográficas y de las fronteras entre las distintas agrupaciones, que se desprende de la vertiente más unitarista de Giné, y que permite a éste explicar la gran variabilidad de los cuadros clínicos en la práctica, aparece la impronta académica del profesor, el catedrático - aunque lo fuese de patología quirúrgica - con obligación de trasmitir a sus alumnos esquemas claros, conforme a la mentalidad nosográfica y anatomoclínica imperante. Ahí surge la necesidad de ofrecer un ordenamiento nosográfico tradicional, categórico, más coherente con los planteamientos de un científico que hace bandera del positivismo y que cree firmemente en la naturaleza orgánica de las enfermedades mentales. No en vano, explica para cada uno de los tres grupos principales de su esquema, las lesiones cerebrales que los sostienen (aunque éstas no hayan servido como criterio para la clasificación); "lesiones de carácter hiperémico" para las locuras, "lesión de nutrición de la sustancia cerebral, reblandecimiento, esclerosis, etc" para las demencias, y "anomalías de conformación ó del desarrollo anatómico del encéfalo" en el caso de los Defectos del desarrollo frénico ${ }^{72}$.

El cuadro en el que plasma su clasificación "para abarcar de un solo golpe de vista" es el siguiente:

\section{CLASIFICACIÓN DE LOS ESTADOS FRENOPÁTICOS.}

\section{ORDEN $10^{\circ}$}

Locuras.- Estado emocional dominante; sin decadencia ni pérdida de las facultades mentales; exageración, depresión ó desorden frenopático; primitivas; susceptibles de pasar al estado crónico ó a la demencia; curables mientras no haya demencia; lesiones cerebrales de caracter hiperémico.

Manía.- Exaltación de la afectividad y de la inteligencia, sin delirio ó con delirio general.

Melancolía.- Predominio de los sentimientos tristes; si hay delirio es general.

Monomanía.-Exaltación de los sentimientos alegres; delirio circunscrito.

Éxtasis.- Suspensión de los actos intelectuales y estéticos, con rigidez general.

Locuras patogenéticas.- Sostenidas por otro estado neuropático o por agentes tóxicos.

72 Ibid. p. 243 


\section{ORDEN $2^{\circ}$.}

Demencias.- Decadencia o pérdida de facultades; primarias o consecutivas; Lesiones de nutrición de la sustancia nerviosa; incurables; sólo susceptible de una terapéutica paliativa que detenga la marcha del proceso necrobiótico.

Parálisis general, ó locura paralítica.- Estado emocional alegre; delirio ambicioso; decadencia y pérdida gradual de las facultades intelectuales y movimientos.

Demencias propiamente dichas.- Caracteres generales del grupo, sin estado emocional particular.

\section{ORDEN $3^{\circ}$.}

Defectos de desarrollo frénico.- De origen congénito; anomalías de conformación o del desarrollo anatómico del encéfalo; incurables; algunas, empero, favorablemente modificables por la educación.

\section{Idiotismo.}

\section{Imbecilidad.}

Niños atrasados.

Inteligencias anormales.

Cretines.

Podemos ver que, aparentemente se respeta una sistemática more botanico, aunque de inmediato se pone de relieve la muy relativa preocupación de Giné por aplicar con algún rigor este método. Tanto en el orden Locuras como en el $2^{\circ}$ orden, Demencias, habla de "clase" donde debería decir "orden"73. No es ésta la única ocasión en que maneja indistintamente los términos "clase", "orden" y "grupo", obviando la consideración, por encima del "orden", de un grado de abstracción nosográfica más amplio agrupable en una "Clase", tal y como quedó establecido en el método clasificatorio de Linneo y en los primeros nosógrafos de la psiquiatría del siglo XVIII. Aquí, aplica un epígrafe genérico para todas las "enfermedades mentales" que es el de "frenopatías", "estados frenopáticos", etc.

En los géneros Manía y Monomanía, Giné aboca a un proceso de atomización nosológica, que, para el segundo caso, es sabido que contribuyó de manera importante, años atras, al declive de la trascendente entidad que introdujera Esquirol.

Algunos de los criterios que apunta para la clasificación de las "especies o formas de la manía" y que resume en un cuadro sinóptico ${ }^{74}$, pueden resultar bastante aleatorios: atendiendo a la marcha (agudísima, aguda, crónica); por la naturaleza del delirio

73 Ibid. p. 243

${ }^{74}$ Ibid. p. 369. 


\section{ANTONIO DIÉGUEZ GÓMEZ}

predominante (alegre, erótica, religiosa, ambiciosa), por el tipo (contínua, remitente, intermitente); por las causas (hereditaria y adquirida), etc. Finalmente se ocupa de dejar claro que el criterio básico en este caso - como en todos los demás - también ha de venir dado por el "concepto nosológico general", según el cuál acepta sólo dos especies o formas a saber: la manía simple (franca, primitiva o idiopática), que tendría como subtipos la manía exaltada y la incoherente, y la manía mixta (híbrida o constitucional). Aquí aparece de nuevo, claramente, la huella de Guislain, pero en la división que posteriormente hace en la manía mixta, estableciendo las variedades razonadora o manía sin delirio de Pinel (locura moral de Prichard y monomanía afectiva de Esquirol) y manía impulsiva ${ }^{75}$, esa huella guislainiana se desvanece al coincidir con claridad el alienista belga únicamente en el reconocimiento de la "manía razonadora de Pinel"76. Junto a esta última Guislain desarrolla un interminable listado de variedades de manía mixta ${ }^{77}$ que en realidad muchas de ellas se corresponden de manera literal con las variedades, tan ramificadas en ese momento, de monomanía en otros autores ${ }^{78}$.

Por otro lado, hemos visto cómo Giné utiliza el delirio como un criterio más de demarcación nosográfica, pero dándole al mismo un estatuto psicopatológico ${ }^{79}$, al contrario de lo que vemos en la obra de Guislain, donde el delirio tiene un claro estatuto nosológico (constituye una de sus seis formas elementales).

Cuando Giné escribe su tratado, la monomanía es ya una categoría nosológica practicamente en desuso ${ }^{80}$. Nuestro autor la mantiene en su clasificación, por más

75 Ibid. p. 375.

76 GUISLAIN, J. (1852), p.126.

77 GiNÉ Y PARTAGÁs, J. (1876), p. 214.

78 Guislain no incluía la monomanía entre sus formas de enfermedad mental. Daba a este término una acepción genérica: cualquier forma de alienación es - para este autor - una monomanía, monofrenia o monofrenopatía. Aunque en su primera obra (Traité des phrénopathies, 1833) Guislain se atiene al sistema de Esquirol (KAHLBAUM, L. -1863-, p. 48.), en sus "Leçons orales", p. 73, es claro que se suma a la corriente crítica al concepto esquiroliano de monomanía ( alimentada, como se sabe, por alienistas tan importantes, como J. P. Falret, Morel, etc. entre otros)

79 Por la ubicación temática que en su obra le otorga Sancho de San Román $(1960$, p. 35.) cabe pensar que comparte también esta idea. BERRIOS, G. y FUENTENEBRO, F., (1996), Delirio. Historia. Clínica. Metateoría. Edit. Trotta. Madrid. p.55., apuntan que durante la segunda mitad del siglo XIX, el contenido del delirio llegaría a ser el criterio más importante para la clasificación y que Guislain fue uno de los más tempranos alienistas en clasificar los delirios de acuerdo al contenido.

${ }^{80}$ El surgimiento y progresiva desaparición de esta especie morbosa de las clasificaciones psiquiátricas, así como la trascendencia epistemológica y social de la misma, ha sido tratada en diversos trabajos. Se pueden consultar los de HUERTAS, R. (1991), prólogo a Memorias sobre la locura y sus variedades. J.E.D. Esquirol. Colección clásicos de la psiquiatría. Edit. Dorsa. Madrid. p. 9-23.; MARTíNEZ PÉREZ, J., (1995) "Problemas científicos y socioculturales en la difusión de una doctrina psiquiátrica: la introducción del concepto de monomanía en España (1821-1864)" en ARQUIOLA, E. Y MARTÍNEZ PÉREZ, J. (coords.) Ciencia en Expansión. Estudios sobre la difusión de las ideas científicas y médicas en España (siglos XVIII-XX). Madrid. Edit. Complutense. pags. 490-520.; más recientemente, del mismo autor, MARTíNEZ-PÉREZ, J., 
que critique la concepción primigenia de Esquirol y en un gesto de pretendida adecuación a los "avances científicos" adopte la que propone Linás en el Dictionnaire des Sciences Médicales $(1871)^{81}$.

En algunos trabajos clásicos, como en el de Peraza de Ayala ${ }^{82}$, se ha apuntado la existencia de un ascendiente de la obra de Pedro Mata sobre la de Giné, amparándose, quizás, en la decidida vocación positivista de ambas figuras médicas. En lo que concierne, al menos, a las propuestas nosográficas de uno y otro autor, esta afirmación está por demostrar. Sería, si acaso, a la hora del estudio de las monomanías, materia en la que sin duda Mata era una referencia obligada en el ámbito español ${ }^{83}$, donde mejor podría reflejarse esa influencia. En este lugar, Giné hace una referencia, que no va más allá del respetuoso cumplido a la obra del pionero de la medicina forense española: "faltaríamos a un deber y a un sentimiento de amor patrio, si al ir a trazar en breve espacio la fenomenología de la monomanía impulsiva, asunto que tanto interesa a la medicina legal, no recomendásemos encarecidamente la lectura de los magníficos trabajos del Dr. D. Pedro Mata"84. Pasa con posterioridad a revisar brevemente cada uno de los subtipos de esta forma de monomanía -impulsiva o instintiva -85 que, salvo en la erotomanía, coinciden uno por uno con los que señala Mata en su $\operatorname{tratado}^{86}$ (m. homicida, m. suicida, $\mathrm{m}$. incendiaria, cleptomanía y dipsomanía) pero que no son otras sino las formas reconocidas desde Marc, hacía ya casi medio siglo. El resto de las formas o especies de monomanía aparecen en el Tratado de Freno-patología ordenadas, según reconoce explícitamente su autor, conforme a la clasificación de Linás, y quedarían de la siguiente manera:

1. Intelectual.

2. Afectiva.

一religiosa (con dos subvariedades, mística y theomanía)

- erótica o erotomanía.

- orgullosa o megalomanía

\footnotetext{
"Catalogando la diversidad del comportamiento humano: la nosología francesa decimonónica ante las conductas delictivas (1800-1855)". Asclepio. vol XLVIII-2-,1996. pags. 87-114.

81 GINÉ Y PARTAGÁs, (1876), pags. 434-436-. Se trata del Dictionnaire Encyclopédique des Sciences Médicales, dirigido por A. Dechambre y editado en París, con una larguísima nómina de figuras de la medicina francesa de esas décadas, como colaboradores.

82 PeRAZA DE AYAla, T., (1947), La psiquiatría española en el siglo XIX. CSIC. Madrid. p. 118.; también en FERRER-HOMBRAVELLA, J. (1948) "Aportaciones a la historia de la psiquiatría española" en Medicina clínica. Tomo XI. $\mathrm{n}^{\circ}$ 6. p. 449.

83 Véase MARTÍNEZ PEREZ, J. (1995), p. 510 y 511.

84 GiNÉ Y PARTAGÁS, J. (1876). p. 452.

85 Ibid. pags. 452-460.

${ }^{86}$ MATA, P. (1857). Tratado de Medicina y Cirugía legal. Teórica y práctica. $3^{\mathrm{a}}$ edic. Carlos Bailly-Bailliere. Madrid. Tomo II. pags. 209-235.
} 


\section{ANTONIO DIÉGUEZ GÓMEZ}

\section{Sensorial o alucinatoria.}

4. Impulsiva o instintiva. ( con las subvariedades ya señaladas).

Otro capítulo en el que Giné adopta directamente el esquema clasificatorio de Guislain es el que concierne a las "especies o formas" de la melancolía ${ }^{87}$, género al que también se refiere repetidamente como Lipemanía, aplicando el viejo neologismo esquiroliano, en vez del término Frenalgia que sería más propio de la fuente referida. Siguiendo literalmente ésta, admite una melancolía general o polimelancolía y un abundante número de melancolías especiales o mono-melancolías (m. sin delirio; $\mathrm{m}$. hipocondríacas, éstas a su vez divididas en dos tipos corporal y mental; m. nostálgica; m. amatoria; m. misantrópica; m. pannofóbica; m. religiosa; m. desesperatoria; $\mathrm{m}$. ansiosa; $\mathrm{m}$. demonofóbica). Como hemos visto para géneros anteriores, la consecuencia del seguimiento tan textual del criterio de Guislain, le lleva de nuevo a una atomización y relativización del valor nosológico y nosográfico de todas estas categorías y subtipos; más aún al finalizar con un tercer epígrafe, el de las melancolías compuestas. Pretende apartarse del guión del alienista belga al hacer mención aparte de la melancolía con estupor y la Locura circular o de doble forma ${ }^{88}$. Ciertamente Guislain incluye esta última e histórica entidad nosológica, acuñada por Falret y Baillarger, entre las melancolías compuestas y aunque la distingue con nitidez de su manía melancólica o de la melancolía maníaca entre otras (según el "fenómeno radical" predominante) ${ }^{89}$, al continuar clasificándola entre las melancolías compuestas - a diferencia de lo que queda registrado en Giné - diluye la singularidad que para la misma estaba comenzando a ser reconocida ${ }^{90}$.

En las clasificaciones francesas de la época91 aparece el Extasis entre las vesanias asociadas a lesiones del movimiento. Allí aparece generalmente junto a otros estados que, en la agrupación de Giné compondrán, como sabemos, otro género aparte - las locuras patogenéticas-. Giné nos remite de inmediato a la raiz básicamente guislainiana de este género - éstasis-, en el que reconoce cuatro especies o formas: místi-

\footnotetext{
87 GiNÉ Y PARTAGÁs, J. (1876). p. 398.

88 Discrepamos en este punto de la apreciación de SANCHO DE SAN ROMAN, (1960), p. 56., quien afirma que Giné incluye la Locura de doble forma entre las melancolías compuestas.

89 El "fenómeno radical" es el criterio de diferenciación diagnóstica básico que utiliza Guislain. Cuando existen por igual síntomas de manía y de melancolía, p. ej., se atiende, para realizar el diagnóstico, a aquellos fenómenos que se presentaron en primer lugar, de manera que si lo hicieron los síntomas melancólicos se tratará de una melancolía maníaca. Giné dice que aunque tomó en cuenta este criterio en sus primeros pasos, con posterioridad lo critica, pues "no dejan de observarse transformaciones completas, por las cuales el estado actual difiere esencialmente del que presentaba la enfermedad en su período de invasión". GINÉ Y PARTAGÁs, J. (1876), p. 242.

90 Y que con escasas modificaciones se mantendrá hasta nuestros dias.

91 Puede verse como ejemplo la de Marcé, en BERCHERIE, P. (1980). p. 56.
} 
co, terrorífico, científico o intelectual y amoroso o erótico, subrayando este último como la variedad observada con mayor frecuencia ${ }^{92}$.

Con la denominación Locuras patogenéticas, Giné reivindica la autoría de un nuevo género que, sin embargo, parece extraído de las enfermedades mentales "sintomáticas" que Baillarger, continuando (a través de Parchappe) la tradición de Georget, separaba de las vesanias puras o esenciales ${ }^{93}$. No encontramos mayor justificación para el reconocimiento de esa originalidad, solo por lo antedicho sino, porque además, por las constantes referencias a Marcé cuando desarrolla cada una de las formas, pone de manifiesto que recoge de manera prácticamente literal la conocida clasificación, que basándose en los autores referidos, el joven Marcé ofrece en su Traité pratique des maladies mentales, publicado en 1862, y que serviría para formar a una generación entera de estudiantes ${ }^{94}$. Establece una división en "dos especies bien distintas por el concepto etiológico": Locuras patogenéticas toxicológicas (alcohólica) y las Locuras patogenéticas sintomáticas de "neurosis" (epiléptica, histérica, coréica y pelagrosa) $)^{95}$.

En el orden Demencias, la sistemática de Giné separa dos géneros: la demencia propiamente dicha por un lado y la Parálisis General de los alienados por otro. Como dejó señalado en su tesis Sancho de San Roman, en el primero de ellos la influencia de Griesinger es determinante ${ }^{6}$. Éste divide en su tratado los "estados de decaimiento intelectual" en dos: 1 . Locura sistematizada, y 2. Demencia propiamente dicha ${ }^{97}$. Tras señalar los reparos que Baillarger y otros autores franceses ponen para aceptar la inclusión de la locura sistematizada entre las demencias, al considerar que "el decaimiento intelectual aquí no es real sino una apariencia resultante del predominio exclusivo de un solo orden de ideas" 98 , Giné justifica la adopción del género propuesto por Griesinger porque "forma una entidad frenopática que establece la transición entre las locuras y las demencias". Las variedades de la Demencia propiamente dicha que recoge Griesinger como demencia agitada y demencia apática, son incorporadas literalmente por Giné. Al margen de éstas, reivindica un lugar singular para la demencia senil, estado al que no le confiere una ubicación nosográfica concreta , pero cabe pensar que lo sitúa dentro del género que estamos tratando por ser éste el lugar en el que con más insistencia trata el problema. Sí deja claro que, en contra de la opinión de Marcé, él considera la demencia senil como una "entidad nosológica particular"99.

\footnotetext{
92 GinÉ y PARTAGÁs, J. (1876), p. 421.

93 BERCHERIE, P., (1980), p. 69.

94 Ibid, pags. $56-57$ y 69.

95 GiNÉ Y PARTAGÁS, J. (1876).p. 461

${ }^{96}$ SANCHO DE SAN ROMAN, R. (1960), p. 61.

97 GRIESINGER, W. (1861), p. 379.

98 GiNÉ Y PARTAGÁs, J. (1876), p. 513.

99 Ibid. p.523.
} 
No hace referencia explícita alguna, - positiva al menos- a otras fuentes, aunque esta entidad no solo aparece en la obra guislainiana con claridad ${ }^{100}$, también está presente desde el "Des maladies mentales" de Esquirol. En efecto el pionero francés ordena de manera nítida la demencia, en su gran texto compilatorio de 1838, en tres variedades: aguda, crónica y senil ${ }^{101}$, aunque al parecer hace esta distinción al menos desde $1814^{102}$. Es la demencia senil de Esquirol el cuadro con el que mayor similitud guarda la breve descripción de esta entidad que Giné realiza en su tratado ${ }^{103}$.

En cuanto al género Locura paralítica o Parálisis general de los alienados, Giné se detiene inicialmente en unas consideraciones que, aunque probablemente ya eran tópicas a esas alturas de la centuria, su mención era obligada al introducir este tema para un médico decididamente partidario de las teorías explicativas de estirpe somaticista. Se trata de la alusión al histórico descubrimiento y demostración por Bayle y Calmeil del sustrato anatomopatologico de la Parálisis General ${ }^{104}$. La división que hace en este género se corresponde con las cuatro variedades descritas por Falret y Linás ${ }^{105}$ : expansiva y melancólica, si predominan los síntomas intelectuales, por un lado, y por otro, parapléjica y congestiva, cuando el predominio es de los síntomas somáticos. Aunque la definición general que Giné propone para este grupo $-2^{\circ}$ orden- es, en definitiva, la de Esquirol, señala que "es preciso segregar la melancolía con estupor" y se suma a la reivindicación de una diferente conceptualización para la demencia aguda de éste, atendiendo a la ausencia de "decadencia frénica". Es la categoría nosológica que Baillarger vincula a la estupidez (Georget) con el nombre de Lipemanía con estupor ${ }^{106}$, incluyéndola en el marco de la Locura (general) propiamente dicha.

Una buena muestra de la ausencia de ingenuidad en el optimismo terapéutico de Giné, al que antes nos referimos, la encontramos en el grupo de los Defectos del desarrollo frénico. Pese a que la incurabilidad es una de sus características definitorias, el que fuera director de Nueva Belén, propone que "cuando el idiotismo - denominación que también utiliza como expresión genérica del grupo - no es muy profundo, todas estas irregularidades del caracter y de la voluntad son notablemente modificables por la educación en asilos apropiados, volviéndose dóciles aquellos que en el seno de la

\footnotetext{
100 GuISLAIN,J. (1852), p. 247.

${ }^{101}$ ESQUIROL, J.E.D., (1838), Des maladies mentales considérées sous les rapports médical, hygiénique et médico-légal. Hemos utilizado la traducción al castellano de D. Raimundo Monasterio y Correa, titulada Tratado completo de las enajenaciones mentales consideradas bajo su aspecto médico, higiénico y legal. Madrid. 1847. Tomo II. p. 96.

102 Berrios, G. (1995), "Dementia". en Berrios, G., PORTER, R., A History of clinical psychiatry. Edit. The Athlone press. Londres. p. 36.

103 GinÉ Y PARTAGÁs, J. (1876), p. 523.

104 Puede verse en BROWN, E.M. (1994), "French Psychiatry Initial Reception of Bayle's Discovery of General Paresis of the Insane". Bulletin of History of Medicine. 68: pags. 235-253.

105 Ibid. p. 532.

106 BERCHERIE, P., (1980), p. 52.
} 
familia eran de todo punto insoportables"107. Parece claro que al decir "educación" se refiere a los medios coercitivos propios del "tratamiento moral". Para este orden suscribe explícitamente las variedades que establece Griesinger: idiotas apáticos o tórpidos y agitados o versátiles, con respecto a lo que él llama idiotas completos. De las formas de idiotismo especificadas en el cuadro general de su clasificación, la huella de Griesinger se confunde en algún punto con la de los franceses, en particular con los estados congénitos de Parchappe y Baillarger ${ }^{108}$. Un ejemplo concreto de ésto puede verse en la vinculación mucho más ténue entre el cretinismo y la degeneración que hay en Giné, quien habla del cretino como "un miembro casi desprendido de la escala antropológica" 109 de la más patente que puede encontrarse en Griesinger -"lo más frecuente es que, el cretinismo, no sea un fenómeno parcial sino la manifestación individual de la degeneración de toda una raza..."110_ o en el propio Morel que identificaba específica y taxativamente esta enfermedad como una manifestación de degeneración ${ }^{111}$.

\section{CONCLUSIÓN: AMBIVALENCIA Y POSIBILISMO}

Para concluir, hacemos una extrapolación que consideramos bien fundamentada. Buena parte de las reflexiones que hemos hecho acerca de las ideas y los planteamientos que sobre nosografía psiquiátrica aparecen en la obra de Giné pueden aplicarse con carácter general al conjunto de su pensamiento psiquiátrico. Hemos atendido a la coexistencia de una visión unitarista de la enfermedad mental, junto con una propuesta ramificadora y categorial de la nosografía, planteamientos generalmente tomados como antagónicos. También hemos visto cómo su defensa del pujante positivismo, resulta muy relativizada e incluso impugnada, a la hora de abordar determinados problemas concretos. De igual manera, sus manifiestos postulados somaticistas quedan claramente subvertidos como resultado del pragmatismo que guia en todo momento la actitud científica, intelectual y profesional de Giné.

Será, en definitiva, el intento de hacer compatibles posiciones teóricas como las referidas, conjugadas con el afán del cientifismo, con actividades e intereses de diversa índole - académicos, ideológicos, mercantilistas, etc - lo que condicione y defina, en última instancia, la realización de Giné.

\footnotetext{
107 GiNÉ Y PARTAGÁS, J. (1852), p. 549.

108 BERCHERIE, P. (1980). p. 56.

109 GINÉ Y PARTAGÁS, J. (1876), p. 560.

110 GRIESINGER, W., (1861), p. 409.

111 Miller, E.,(1995). “ Mental Retardation”, en Berrios, G., PORTER, R., A History of clinical psychiatry, Edit. The Athlone press. Londres. p. 212.
} 